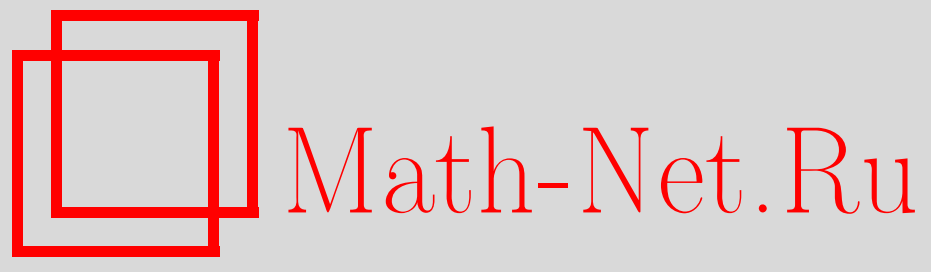

М. А. Ольшанецкий, Эллиптическая гидродинамика и квадратичные алгебры векторных полей на торе, $T M \Phi$, 2007, том 150, номер 3, 355-370

DOI: https://doi.org/10.4213/tmf5984

Использование Общероссийского математического портала Math-Net.Ru подразумевает, что вы прочитали и согласны с пользовательским соглашением http://www . mathnet.ru/rus/agreement

Параметры загрузки:

IP : 54.162 .27 .143

26 апреля 2023 г., 12:32:33

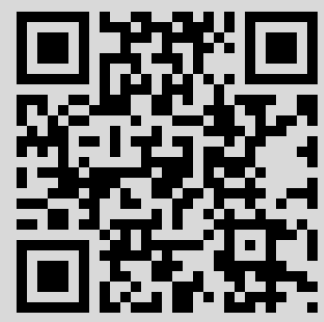




\title{
ЭЛЛИПТИЧЕСКАЯ ГИДРОДИНАМИКА И КВАДРАТИЧНЫЕ АЛГЕБРЫ ВЕКТОРНЫХ ПОЛЕЙ НА ТОРЕ
}

\begin{abstract}
Построена квадратичная алгебра Пуассона на гамильтоновых функциях на двумерном торе, которая согласована с канонической пуассоновой структурой. Эта алгебра представляет собой бесконечномерное обобщение классических алгебр Склянина-Фейгина-Одесского и приводит к интегрируемой модификации двумерной гидродинамики идеальной жидкости на торе. Гамильтониан стандартной двумерной гидродинамики задается оператором Лапласа и потому зависит от метрики. Новый гамильтониан, полученный при замене оператора Лапласа на зависящий от комплексной структуры псевдодифференциальный эллиптический оператор, оказывается представителем коммутативной бигамильтоновой иерархии. В заключение строится биалгеброид Ли векторных полей на торе.
\end{abstract}

Ключевые слова: уравнение Эйлера гидродинамики идеальной жидкости, квадратичные алгебры Пуассона.

\section{1. ВВЕДЕНИЕ}

Гидродинамика идеальной несжимаемой жидкости на двумерной поверхности, как известно, представляет собой неинтегрируемую систему. Даже 4-вихревое приближение динамики идеальной жидкости оказывается неинтегрируемым [1]. В работе [2] нами были описаны интегрируемые версии гидродинамики на двумерном торе. Классическое уравнение Эйлера движения идеальной жидкости имеет вид

$$
\partial_{t} \mathbf{S}=\left\{\mathbf{S}, \Delta^{-1} \mathbf{S}\right\}
$$

где $\mathbf{S}$ - функция завихренности потока жидкости, $\{\cdot, \cdot\}-$ каноническая скобка Пуассона и $\Delta$ - оператор Лапласа. Модифицированная и эллиптическая гидродинамики определены на эллиптической кривой, т.е. на торе с фиксированной комплексной структурой. Соответствующие уравнения записываются как

$$
\partial_{t} \mathbf{S}=\left\{\mathbf{S}, \bar{\partial}^{-2} \mathbf{S}\right\}, \quad \partial_{t} \mathbf{S}=\{\mathbf{S}, \wp(\bar{\partial}) \mathbf{S}\}
$$

* Институт теоретической и экспериментальной физики, Москва, Россия. E-mail: olshanet@itep.ru 
где $\bar{\partial}$ - оператор, зависящий от комплексной структуры, а ю- функция Вейерштрасca.

Такая модификация оператора инерции жидкости, в которой оператор Лапласа $\Delta=\partial \bar{\partial}$, зависящий от метрики на $T^{2}$, заменяется на зависящие от комплексной структуры на $T^{2}$ операторы $\bar{\partial}^{2}$ или $\wp(\bar{\partial})^{-1}$, приводит к интегрируемости и даже к бигамильтоновости соответствующей системы. Оказывается, что при этом возникает бесконечное семейство интегралов движения в инволюции относительно двух скобок Пуассона. Одна из скобок представляет собой стандартную линейную скобку Пуассона-Ли на пространстве, дуальном к алгебре бездивергентных векторных полей на $T^{2}$. Другая пуассонова структура есть квадратичная алгебра Пуассона на гамильтонианах векторных полей. Эти две скобки оказываются совместными и задаются одной и той же классической $r$-матрицей. Квадратичная пуассонова алгебра представляет собой при этом бесконечномерный аналог классической алгебры Склянина-Фейгина-Одесского [3]. Более того, существует последовательность коммутирующих гамильтонианов, подчиняющихся этой линейно-квадратичной бигамильтоновой структуре иерархии эллиптической гидродинамики, которая тем самым проявляет интегрируемость в сильнейшей форме.

Согласованные скобки Пуассона приводят к построению биалгеброида Ли [4] над пространством гамильтонианов на торе. Скобки, канонически определенные на сечениях биалгеброида, приводят к квадратичным алгебрам векторных полей на двумерном торе. Можно описать структуру биалгеброида в терминах бесконечномерного аналога классической эллиптической матрицы типа Белавина-Дринфельда [5].

Раздел 3 базируется на результатах, полученных в работе [2]. В этой же работе можно найти доказательства утверждений.

\section{2. ДВУМЕРНАЯ ГИДРОДИНАМИКА ИДЕАЛЬНОЙ ЖИДКОСТИ}

2.1. Алгебра Ли векторных полей. Пусть $M$ - двумерная ориентируемая поверхность с заданной на ней два-формой $\omega$. Рассмотрим векторное поле $\mathbf{v}=$ $V^{\alpha} \partial_{\alpha}, \alpha=1,2$, на $M$ и обозначим через $\mathcal{L}_{\mathbf{v}}=d \iota_{\mathbf{v}}+\iota_{\mathbf{v}} d$ производную Ли, где $\iota_{\mathbf{v}}-$ оператор свертки. Дивергенция $\operatorname{div}(\mathbf{v})$ поля $\mathbf{v}$ представляет собой два-форму $d \iota_{\mathbf{v}} \omega$, или, локально,

$$
\operatorname{div}(\mathbf{v})=\epsilon^{\gamma \beta} \partial_{\gamma} V^{\alpha} \omega_{\alpha \beta} .
$$

Векторное поле $\mathbf{v}$ называется бездивергентным, если $\operatorname{div}(\mathbf{v})=0$. Поскольку дваформа $\omega$ замкнута, $d \omega=0$, бездивергентность $\mathbf{v}$ означает, что векторное поле сохраняет форму, $\mathcal{L}_{\mathbf{v}} \omega=0$. Бездивергентные векторные поля на $M$ образуют алгебру Ли:

$$
\operatorname{SVect}(M)=\left\{\mathbf{v} \mid \mathcal{L}_{\mathbf{v}} \omega=0, \quad V^{\alpha} \in C^{\infty}(M)\right\}
$$

Из (2.1) следует, что локально существует функция $\psi_{\mathbf{v}}$ такая, что $\iota_{\mathbf{v}} \omega=d \psi_{\mathbf{v}}$ или $V^{\alpha} \omega_{\alpha \beta}=\partial_{\beta} \psi$. Функция $\psi_{\mathbf{v}}$ называется функцией потока. Глобально можно добавить к $d \psi_{\mathbf{v}}$ замкнутую один-форму $\alpha \in H^{1}(M), d \alpha=0, \alpha \neq d f$. Векторное поле называется гамильтоновым, если один-форма $\iota_{\mathbf{v}} \omega$ точна. Обозначим через 
$\operatorname{SVect}^{\mathrm{h}}(M)$ подпространство гамильтоновых векторных полей и через $\operatorname{Ham}(M)=$ $\{\psi\}$ - пространство гладких функций потока. Пространства $\operatorname{SVect}(M)$ и $\operatorname{Ham}(M)$ входят в точную последовательность

$$
0 \rightarrow \mathbb{C} \rightarrow \operatorname{Ham}(M) \rightarrow \operatorname{SVect}(M) \rightarrow H^{1}(M) \rightarrow 0
$$

Пусть $\left\{\gamma_{a}, a=1, \ldots, 2 g\right\}$ - базис одномерных гомологий $H_{1}(M)$. Тогда отображение $\operatorname{SVect}(M) \rightarrow H^{1}(M)$ задается потоками

$$
\mathbf{v} \rightarrow \int_{\gamma_{a}} \iota_{\mathbf{v}} \omega
$$

Таким образом, $\operatorname{SVect}(M) / \operatorname{SVect}^{\mathrm{h}}(M) \sim H^{1}(M)$ и $\operatorname{SVect}^{\mathrm{h}}(M) \sim \operatorname{Ham}(M) / \mathbb{C}=$ $\operatorname{Ham}^{0}(M)$. В дальнейшем рассматривается $\operatorname{Ham}^{0}(M)$.

Пространства $\operatorname{Ham}(M)$ и $\operatorname{Ham}^{0}(M)$ являются алгебрами Ли по отношению к скобкам Пуассона

$$
\left\{\psi_{\mathbf{v}}, \psi_{\mathbf{u}}\right\}=\iota_{\mathbf{u}} \iota_{\mathbf{v}} \omega
$$

Имеют место следующие соотношения между алгебрами $\operatorname{SVect}^{\mathrm{h}}(M)$ и $\operatorname{Ham}(M)$ :

$$
\left\{\psi_{\mathbf{u}}, \psi_{\mathbf{v}}\right\}=\psi_{[\mathbf{v}, \mathbf{u}]}+c(\mathbf{v}, \mathbf{u})
$$

где $c(\mathbf{v}, \mathbf{u})$ - центральное расширение $\operatorname{SVect}(M)$. Чтобы доказать это, рассмотрим следующую цепочку тождеств:

$$
\iota_{[\mathbf{v}, \mathbf{u}]} \omega=\mathcal{L}_{\mathbf{v}} \iota_{\mathbf{u}} \omega=\left(\iota_{\mathbf{v}} d+d \iota_{\mathbf{v}}\right) \iota_{\mathbf{u}} \omega=d \iota_{\mathbf{v}} \iota_{\mathbf{u}} \omega
$$

Отсюда следует, что $d \psi_{[\mathbf{v}, \mathbf{u}]}=d\left\{\psi_{\mathbf{v}}, \psi_{\mathbf{u}}\right\}$. Разность $\psi_{[\mathbf{v}, \mathbf{u}]}-\left\{\psi_{\mathbf{v}}, \psi_{\mathbf{u}}\right\}$ тем самым равна константе $c(\mathbf{v}, \mathbf{u})$. Условие коцикла следует из тождеств Якоби для алгебры Пуассона и для алгебры Ли векторных полей.

Определим коалгебру Ли обобщенных функций на $\operatorname{Ham}^{0}(M)$ :

$$
\operatorname{Ham}^{0 *}(M)=\left\{\mathbf{S} \mid\langle\psi, \mathbf{S}\rangle:=\int_{M} \omega(\psi \mathbf{S})<\infty\right\} .
$$

Лемма. Коприсоединенное действие $\operatorname{Ham}^{0}(M)$ на $\operatorname{Ham}^{0 *}(M)$ имеет вид

$$
\operatorname{ad}_{\psi}^{*} \mathbf{S}=-\{\psi, \mathbf{S}\}
$$

ДоКАЗАТЕЛЬСТво. С помощью прямых вычислений получим

$$
\int_{M}\left\{\psi_{\mathbf{v}} \psi_{\mathbf{u}}\right\} \mathbf{S} \omega=\int_{M} U^{\alpha} \partial_{\alpha} \psi_{\mathbf{v}} \mathbf{S} \omega=-\int_{M} \psi_{\mathbf{v}} U^{\alpha}\left(\partial_{\alpha} \mathbf{S}\right) \omega-\int_{M} \psi_{\mathbf{v}} \mathbf{S} \partial_{\alpha}\left(U^{\alpha} \omega\right) .
$$

Первый член представляет собой искомые скобки Пуассона, а второй член обращается в нуль в силу (2.1). 
Коалгебра Ли $\operatorname{Ham}^{0 *}(M)$ снабжена стандартными линейными скобками Пуассона-Ли. Пусть $\nabla f(\mathbf{S}) \in \operatorname{Ham}^{0}(M)$ - градиент функционала $f$. Тогда скобка Пуассона-Ли принимает вид

$$
\{f(\mathbf{S}), g(\mathbf{S})\}=\int_{M} \omega\{\nabla f(\mathbf{S}), \nabla g(\mathbf{S})\} \mathbf{S} .
$$

Она вырожденна на $\operatorname{Ham}^{0 *}(M)$ и обладает бесконечным набором функционалов Казимира. Например, в плоском случае

$$
C_{k}=\int_{M} \omega \mathbf{S}^{k}
$$

Однако на коприсоединенных орбитах группы $\operatorname{SDiff}(M)$ диффеоморфизмов, сохраняющих форму $\omega$, скобка не вырожденна.

2.2. Уравнения Эйлера. Пусть $\mathbf{v}$ - бездивергентное векторное поле, а $p$ давление. В плоском пространстве $M$ уравнение Эйлера для $\mathbf{v}$ имеет следующий вид [6]:

$$
\partial_{t} \mathbf{v}=-(\mathbf{v} \cdot \nabla) \mathbf{v}-\operatorname{grad} p .
$$

Для завихренности $\operatorname{rot} \mathbf{v}$ его можно переписать как

$$
\partial_{t} \operatorname{rot} \mathbf{v}=\operatorname{rot}(\mathbf{v} \times \operatorname{rot} \mathbf{v})
$$

В двумерном случае $\mathbf{S}=\operatorname{rot} \mathbf{v}=\partial_{1} V^{2}-\partial_{2} V^{1}$ представляет собой функцию на $M$, а выражение (2.6) принимает вид

$$
\partial_{t} \mathbf{S}=\{\mathbf{S}, \psi\}
$$

где $\psi$ - функция потока $\left(\partial_{1} \psi=-V^{2}, \partial_{2} \psi=V^{1}\right)$. Из определений полей $\psi_{\mathbf{v}}$ и $\mathbf{S}(\mathbf{v})$ следует, что

$$
\mathbf{S}=\Delta \psi
$$

где $\Delta$ - оператор Лапласа.

Рассмотрим теперь двумерную поверхность $M$ с римановой метрикой

$$
g=g_{\alpha \beta} d x^{\alpha} d x^{\beta}
$$

задающей отображение $\mathcal{C}$ векторных полей $\left\{\mathbf{v}=V^{\alpha} \partial_{\alpha}\right\}$ на $M$ в пространство одинформ:

$$
\mathcal{C}: \Gamma \rightarrow \Omega^{1}(M), \quad \mathcal{C}(\mathbf{v})=V^{\alpha} g_{\alpha \beta} d x^{\beta} .
$$

Пусть $*$ - операция Ходжа на $M$ по отношению к форме объема $\omega=\sqrt{\operatorname{det} g} d x^{1} \wedge d x^{2}$. Операция rot представляет собой отображение

$$
\operatorname{rot}: \Gamma \rightarrow C^{\infty}(M), \quad \mathbf{S}=\operatorname{rot} \mathbf{v}=* d \mathcal{C}(\mathbf{v})=\frac{1}{\sqrt{\operatorname{det} g}} \epsilon^{\gamma \beta} \partial_{\gamma} V^{\alpha} g_{\alpha \beta} .
$$


Напомним, что функция потока задается соотношением $d \psi_{\mathbf{v}}=\iota_{\mathbf{v}} \omega$. Из выражения (2.8) следует, что функция потока и завихренность связаны тем же соотношением (2.7) с оператором $\Delta$, замененным на оператор Бельтрами-Лапласа на $M$ :

$$
\Delta=\frac{1}{\sqrt{\operatorname{det} g}} \partial_{a} g^{a b} \sqrt{\operatorname{det} g} \partial_{b} .
$$

Из соотношения (2.7) следует, что $\mathbf{S} \in \operatorname{Ham}^{0 *}(M)$.

Уравнение Эйлера на произвольном римановом многообразии $M$ имеет вид

$$
\partial_{t} \mathbf{S}=\left\{\mathbf{S}, \Delta^{-1} \mathbf{S}\right\}=\frac{1}{\operatorname{det}^{1 / 2} g}\left(\partial_{1} \mathbf{S} \partial_{2} \Delta^{-1} \mathbf{S}-\partial_{1} \Delta^{-1} \mathbf{S} \partial_{2} \mathbf{S}\right)
$$

и, таким образом, определено на коалгебре $\operatorname{Ham}^{0 *}(M)$ и зависит от метрики $g$ на $M$. Оно имеет гамильтонов вид по отношению к скобкам (2.4) на $\operatorname{Ham}^{0 *}(M)$ с гамильтонианом

$$
H=-\frac{1}{2} \int_{M}\left(\Delta^{-1} \mathbf{S}\right) \mathbf{S} \omega
$$

2.3. Волчок Эйлера-Арнольда. Уравнение Эйлера можно привести к общему виду, называемому уравнением волчка Эйлера-Арнольда [6], [7].

Пусть $G$ - группа Ли, а $\mathfrak{g}$ - ее алгебра Ли. Рассмотрим обратимый линейный оператор $\mathbf{J}$, который отображает коалгебру $\mathfrak{g}^{*}$ в $\mathfrak{g}$. Обратный к нему оператор $\mathbf{J}^{-1}$ называется тензором инерции. Волчок Эйлера-Арнольда, отвечающий группе $G$, представляет собой гамильтонову систему на $\mathfrak{g}^{*}$ по отношению к линейным скобкам Пуассона-Ли на $\mathfrak{g}^{*}$ с гамильтонианом, задаваемым квадратичной формой:

$$
H=-\frac{1}{2}\langle\mathbf{S}, \mathbf{J}(\mathbf{S})\rangle, \quad \mathbf{S} \in \mathfrak{g}^{*},
$$

где через $\langle\cdot, \cdot\rangle$ обозначено спаривание между $\mathfrak{g}$ и $\mathfrak{g}^{*}$. Градиент $\nabla H$ можно рассматривать, как элемент $\mathfrak{g}$, а соответствующие гамильтоновы уравнения движения принимают при этом вид

$$
\partial_{t} \mathbf{S}=\{H, \mathbf{S}\}:=\operatorname{ad}_{\nabla H}^{*} \mathbf{S}, \quad \nabla H=\mathbf{J}(\mathbf{S})
$$

Напомним, что скобки Пуассона-Ли вырожденны на $\mathfrak{g}^{*}$, а их симплектические листы суть коприсоединенные орбиты группы $G$. Чтобы оказаться на определенной коприсоединенной орбите $\mathcal{O}$, надо зафиксировать значения операторов Казимира для линейной скобки.

В случае двумерной гидродинамики $G \subset \operatorname{SDiff}(M)$, гамильтониан (2.11) представляет собой функционал на коалгебре Ли $\operatorname{Ham}^{0 *}(M)$, а тензор инерции задается оператором Бельтрами-Лапласа $\mathbf{J}^{-1}=\Delta: \operatorname{Ham}^{0}(M) \rightarrow \operatorname{Ham}^{0 *}(M)(2.9)$, зависящим от метрики $g$. Из леммы следует, что правая часть уравнения движения (2.10) принимает вид коприсоединенного действия (см. (2.13)). 


\section{3. ЭЛЛИПТИЧЕСКАЯ ГИДРОДИНАМИКА}

При специальном выборе $\mathbf{J}$ система Эйлера-Арнольда становится вполне интегрируемой. Зафиксируем комплексную структуру на $M$.

ПреДПоЛожениЕ. Существует мероморфная функиия $f(z)$ на $M$ такая, что волчок Эйлера-Арнолъда на $\operatorname{SDiff}(M)$ становится интегрированным для обратного оператора инериии $\mathbf{J}$, определенного как псевдодифберенциалъный оператор $f(\bar{\partial})$ :

$$
\psi(x)=\mathbf{J}(\mathbf{S})(x)=f(\bar{\partial}) \mathbf{S}(x), \quad x \in M .
$$

Докажем эту гипотезу для двумерного тора, выбирая $f(z)=\wp(z)-$ функции Вейерштрасса.

3.1. Алгебра Пуассона-Ли на торе. Рассмотрим двумерный тор

$$
M=T^{2}=\mathbb{C} /(\mathbb{Z} \oplus \mathbb{Z}), \quad T^{2}=\left\{\left(x_{1}, x_{2}\right), 0<x_{j} \leqslant 1\right\} .
$$

Пусть

$$
\widetilde{\mathbb{Z}}^{(2)}=\left\{\alpha=\left(\alpha_{1}, \alpha_{2}\right), \alpha_{j} \in \mathbb{Z}, \alpha \neq(0,0)\right\}
$$

- двумерная решетка с выколотым началом отсчета и

$$
T_{\alpha}=\mathbf{e}(\alpha \cdot x)=e^{2 \pi i\left(\alpha_{1} x_{1}+\alpha_{2} x_{2}\right)}, \quad \alpha \in \widetilde{\mathbb{Z}}^{(2)},
$$

- базис Фурье в алгебре Ли функций потока:

$$
\operatorname{Ham}^{0}\left(T^{2}\right)=\left\{\psi(x)=\left.\sum_{\alpha \in \widetilde{\mathbb{Z}}^{(2)}} c_{\alpha} T_{a}\left|\sup _{\alpha}\left(1+\alpha_{1}^{2}+\alpha_{2}^{2}\right)^{k}\right| c_{\alpha}\right|^{2}<\infty, \quad k \in \mathbb{N}\right\} .
$$

Скобка Ли представляет собой просто каноническую скобку Пуассона на $T^{2}$. В базисе Фурье она принимает вид

$$
\left[T_{\alpha}, T_{\beta}\right]=(\alpha \times \beta) T_{\alpha+\beta}, \quad\left(\alpha \times \beta=\alpha_{1} \beta_{2}-\alpha_{2} \beta_{1}\right) .
$$

Коалгебра $\operatorname{Ham}^{0 *}\left(T^{2}\right)$ задается пространством обобщенных функций на $\operatorname{Ham}^{0}\left(T^{2}\right)$,

$$
\operatorname{Ham}^{0 *}\left(T^{2}\right)=\left\{\mathbf{S} \mid\langle\psi, \mathbf{S}\rangle=\int_{T^{2}} d x_{1} d x_{2}(\psi \mathbf{S})<\infty, \quad \psi \in \operatorname{Ham}^{0}\left(T^{2}\right)\right\} .
$$

Структура Пуассона-Ли на $\operatorname{Ham}^{0 *}\left(T^{2}\right)$ имеет канонический вид

$$
\left\{\mathbf{S}, \mathbf{S}^{\prime}\right\}_{1}=\partial_{x_{1}} \mathbf{S} \partial_{x_{2}} \mathbf{S}^{\prime}-\partial_{x_{1}} \mathbf{S}^{\prime} \partial_{x_{2}} \mathbf{S}
$$

или

$$
\left\{S_{\alpha}, S_{\beta}\right\}_{1}=(\alpha \times \beta) S_{\alpha+\beta},
$$

где $\mathbf{S}=\sum_{\alpha \in \widetilde{\mathbb{Z}}^{(2)}} \mathbf{S}_{\alpha} T_{\alpha}$. Нижний индекс 1 означает, что рассматриваются линейные скобки. 
3.2. Эллиптическая модификация двумерной гидродинамики. Введем комплексную структуру на $T^{2}$. Пусть $\epsilon=\left(\epsilon_{1}, \epsilon_{2}\right), 0<\epsilon_{j}<1$, - два иррациональных числа. Рассмотрим комплексную координату на $T^{2}$

$$
Z=-\frac{2 \pi i}{\tau-\bar{\tau}}\left(\frac{\tau x_{1}}{\epsilon_{1}}-\frac{x_{2}}{\epsilon_{2}}\right), \quad \bar{Z}=-\frac{2 \pi i}{\tau-\bar{\tau}}\left(\frac{\bar{\tau} x_{1}}{\epsilon_{1}}-\frac{x_{2}}{\epsilon_{2}}\right),
$$

и соответствующий оператор $\partial_{\bar{Z}}=\bar{\partial}_{\epsilon, \tau}$. Заметим, что он действует на фурьегармониках как $\bar{\partial}_{\epsilon, \tau} \mathbf{e}(\alpha \cdot x)=\left(\epsilon_{1} \alpha_{1}+\epsilon_{2} \alpha_{2} \tau\right) \mathbf{e}(\alpha \cdot x)$. Обозначим через $E_{\tau}$ тор $T^{2}$ с введенными комплексными кооодинатами.

Определим обратный оператор инерции $\mathbf{J}: \operatorname{Ham}^{0 *}\left(T^{2}\right) \rightarrow \operatorname{Ham}^{0}\left(T^{2}\right)$,

$$
\mathbf{J}: S_{\alpha} \rightarrow \psi_{\alpha}=\wp_{\epsilon}(\alpha) S_{\alpha}, \quad \wp_{\epsilon}(\alpha)=\wp\left(\epsilon_{1} \alpha_{1}+\epsilon_{2} \alpha_{2} \tau ; \tau\right),
$$

где $\alpha \in \widetilde{\mathbb{Z}}^{(2)}($ см. (3.1)) и

$$
\wp(u ; \tau)=\frac{1}{u^{2}}+\sum_{j, k}^{\prime}\left(\frac{1}{(j+k \tau+u)^{2}}-\frac{1}{(j+k \tau)^{2}}\right), \quad u \in E_{\tau},
$$

- функция Вейерштрасса на $E_{\tau}$. Она представляет собой дважды периодическую функцию на $E_{\tau}$ с полюсом второго порядка в точке $u=0$. Оператор хорошо определен, поскольку периоды $\epsilon_{j}$ иррациональны. Другими словами, оператор $\mathbf{J}$ представляет собой псевдодифференциальный оператор на $T^{2}$ :

$$
\mathbf{J}: \mathbf{S}(x) \rightarrow \wp\left(\bar{\partial}_{\epsilon, \tau}\right) \mathbf{S}(x)
$$

Уравнения движения задаются соответствующим квадратичным гамильтонианом (2.12)

$$
H=-\frac{1}{2} \int_{T^{2}} \mathbf{S} \wp\left(\bar{\partial}_{\epsilon, \tau}\right)(\mathbf{S})=-\frac{1}{2} \sum_{\alpha \in \widetilde{\mathbb{Z}}^{(2)}} \wp_{\epsilon}(\alpha) S_{\alpha} S_{-\alpha}
$$

по отношению к линейным скобкам Пуассона на $\operatorname{Ham}^{0 *}\left(T^{2}\right)$ :

$$
\left.\partial_{t} \mathbf{S}=\left\{\mathbf{S}, \wp\left(\bar{\partial}_{\epsilon, \tau}\right) \mathbf{S}\right)\right\}_{1}
$$

В фурье-компонентах эти скобки имеют вид

$$
\partial_{t} S_{\alpha}=\sum_{\gamma \in \widetilde{\mathbb{Z}}^{(2)}} S_{\gamma} \wp_{\epsilon}(\alpha) S_{\alpha-\gamma}
$$

Будем называть эти уравнения эллиптической гидродинамикой на торе $T^{2}$.

3.3. Представление Лакса и $r$-матрица. Пусть

$$
\vartheta(z ; \tau)=q^{1 / 8} \sum_{n \in \mathbb{Z}}(-1)^{n} e^{\pi i(n(n+1) \tau+2 n z)}
$$


представляет собой нечетную тета-функцию, определенную эллиптической кривой $\widetilde{E}_{\tau}=\mathbb{C} /(\mathbb{Z}+\tau \mathbb{Z})^{1)}$. Рассмотрим на $E_{\tau} \times \widetilde{E}_{\tau}$ функции

$$
\begin{gathered}
\phi(u, z)=\frac{\vartheta(u+z) \vartheta^{\prime}(0)}{\vartheta(u) \vartheta(z)}, \quad u \in E_{\tau}, \quad z \in \widetilde{E}_{\tau} ; \\
\varphi_{\epsilon}(\alpha, z)=\mathbf{e}\left(\alpha_{2} z\right) \phi\left(\epsilon_{1} \alpha_{1}+\tau \epsilon_{2} \alpha_{2}, z\right), \quad \mathbf{e}\left(\alpha_{2} z\right)=e^{2 \pi i\left(\alpha_{2} z\right)} .
\end{gathered}
$$

Из свойств $\vartheta(z ; \tau)$ следует, что

$$
\varphi_{\epsilon}(\gamma, z+1)=\mathbf{e}\left(\gamma_{2}\right) \varphi_{\epsilon}(\gamma, z), \quad \varphi_{\epsilon}(\gamma, z+\tau)=\mathbf{e}\left(-\gamma_{1}\right) \varphi_{\epsilon}(\gamma, z)
$$

Определим оператор Лакса $f(x) \rightarrow\{L, f\}:$

$$
L(x ; z)=-\sum_{\alpha \in \widetilde{\mathbb{Z}}^{(2)}} S_{\alpha} \varphi_{\epsilon}(\alpha, z) T_{\alpha}, \quad T_{\alpha}=\mathbf{e}(\alpha \cdot x)=e^{2 \pi i\left(\alpha_{1} x_{1}+\alpha_{2} x_{2}\right)},
$$

с действием Пуассона на пространстве гладких функций $\left\{f(x), x \in T^{2}\right\}$. Здесь $z \in \widetilde{E}_{\tau}-$ спектральный параметр. На фазовом пространстве $\mathcal{R}^{0}$ эллиптической гидродинамики (см. ниже $(3.14))$ оператор $L(x, z)$ хорошо определен при $z \in \widetilde{E}_{\tau}$, $z \neq 0$.

ПреДЛОЖЕНИЕ 1. Уравнения движения (3.7) допускают бездисперсионное представление Лакса

$$
L=\{L, M\}_{1},
$$

где L задается выражением (3.9) и

$$
M(x ; z)=-\sum_{\alpha \in \widetilde{\mathbb{Z}}^{(2)}} S_{\alpha} f_{\epsilon}(\alpha, z) \mathbf{e}(\alpha \cdot x),
$$

при этом

$$
f_{\epsilon}(\alpha, z)=f\left(\epsilon_{1} \alpha_{1}+\tau \epsilon_{2} \alpha_{2}, z\right)=\left.\mathbf{e}\left(\epsilon_{2} \alpha_{2}\right) \partial_{u} \phi(u, z)\right|_{u=\epsilon_{1} \alpha_{1}+\tau \epsilon_{2} \alpha_{2}} .
$$

Из (3.8) видно, что оператор $L$ удовлетворяет условиям квазипериодичности:

$$
\begin{aligned}
& L\left(x_{1}, x_{2} ; z\right)=L\left(x_{1}+\epsilon_{2}, x_{2} ; z+1\right), \\
& L\left(x_{1}, x_{2} ; z\right)=L\left(x_{1}, x_{2}-\epsilon_{1} ; z+\tau\right) .
\end{aligned}
$$

Отсюда следует, что интегралы моментов $\int_{T^{2}}(L(x, z))^{k}$ оказываются дваждыпериодическими функциями на базисной спектральной кривой $\widetilde{E}_{\tau}$. Тем самым они могут быть разложены по базису функции Вейерштрасса и ее производных:

$$
\int_{T^{2}}(L(x, z))^{k}=I_{0, k}+\sum_{s=2}^{k} I_{s, k} \wp^{(s-2)}(z) .
$$

\footnotetext{
1) Здесь $\widetilde{E}_{\tau}-$ базисная спектральная кривая. Не следует ее путать с $E_{\tau}$.
} 


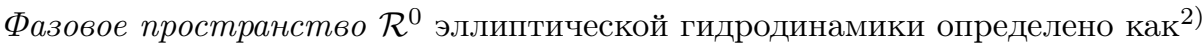

$$
\mathcal{R}^{0}=\left\{\mathbf{S} \in \operatorname{Ham}^{0 *}\left(T^{2}\right) \mid I_{s, j}<\infty, \quad \lim _{j \rightarrow \infty} \frac{I_{s, j+1}(\mathbf{S})}{I_{s, j}(\mathbf{S})}<1, \quad s \leqslant j\right\} .
$$

Определим $r$-матрицу на $T^{2} \otimes T^{2}$ с помощью соотношения

$$
r_{\epsilon, \tau}(x, y ; z)=\sum_{\alpha \in \widetilde{\mathbb{Z}}^{(2)}} \varphi_{\epsilon}(\alpha, z) \mathbf{e}(\alpha \cdot x) \mathbf{e}(-\alpha \cdot y), \quad z \in \widetilde{E}_{\tau}, \quad x, y \in T^{2} .
$$

ПреДЛОЖЕНИЕ 2. 1. r-Матрица (3.15) удовлетворяет “бездисперсионному" уравнению Янга-Бакстера:

$$
\begin{aligned}
& \left\{r_{\epsilon, \tau}(x, y ; z-w), r_{\epsilon, \tau}(x, v ; z)\right\}_{1}+\left\{r_{\epsilon, \tau}(x, y ; z-w), r_{\epsilon, \tau}(y, v ; w)\right\}_{1}+ \\
& \quad+\left\{r_{\epsilon, \tau}(x, v ; z), r_{\epsilon, \tau}(y, v ; w)\right\}_{1}=0 .
\end{aligned}
$$

2. Каноническая скобка на $T^{2}$ в терминах оператора Лакса (3.9) принимает вид

$$
\{L(x ; z), L(y ; w)\}_{1}=\left\{r_{\epsilon, \tau}(x, y, z-w), L(x ; z)\right\}_{1}+\left\{r_{\epsilon, \tau}(x, y, z-w) L(y ; w)\right\}_{1} .
$$

Форма скобок (3.17) предполагает, что

$$
\left\{\int_{T^{2}}(L(x, z))^{k}, \int_{T^{2}}(L(x, z))^{j}\right\}_{1}=0 .
$$

В свою очередь, соотношение (3.13) генерирует бесконечный набор законов сохранения $I_{s, k}$, находящихся в инволюции. Они задают иерархию коммутирующих потоков:

$$
\partial_{s, k} \mathbf{S}=\left\{I_{s, k}, \mathbf{S}\right\}_{1} .
$$

Эти уравнения можно представить в виде бездисперсионных уравнений Лакса с соответствующими операторами $M_{s, k}$.

3.4. Квадратичная алгебра Пуассона и бигамильтонова структура на $\operatorname{SDiff}\left(T^{2}\right)$. Оказывается, что иерархию (3.18) можно описать квадратичной скобкой Пуассона на том же фазовом пространстве. Для этого перейдем сначала от оператора Лакса $L$ алгебры Ли к оператору, содержащему добавочный константный член

$$
\tilde{L}(x ; z)=-S_{0}+L(x ; z) .
$$

Зададим квадратичную алгебру Пуассона формулой

$$
\{\tilde{L}(x ; z), \tilde{L}(y ; w)\}_{2}=\left\{r_{\epsilon, \tau}(x, y ; z-w), \tilde{L}(x ; z) \tilde{L}(y ; w)\right\}_{1} .
$$

Свойство Якоби обеспечивается классическим уравнением Янга-Бакстера (3.16). Для построения алгебры следует убрать зависимость от спектрального параметра из формулы (3.20).

\footnotetext{
${ }^{2)}$ Из этих условий следует существование специальных классов сохраняющихся зарядов, описанных в предложении 3.5 работы [2].
} 
ПреДЛОЖЕНИЕ 3. В терминах фуръе-мод алгебра Пуассона (3.20) имеет вид

$$
\begin{gathered}
\left\{S_{\alpha}, S_{0}\right\}_{2}=\sum_{\gamma \neq \alpha} S_{\alpha-\gamma} S_{\gamma} \wp_{\epsilon}(\gamma)(\alpha \times \gamma), \\
\left\{S_{\alpha}, S_{\beta}\right\}_{2}=S_{0} S_{\alpha+\beta}(\alpha \times \beta)+\sum_{\gamma \neq \alpha,-\beta} S_{\alpha-\gamma} S_{\beta+\gamma} \mathbf{f}_{\epsilon}(\alpha, \beta, \gamma)(\gamma \times(\alpha-\beta)),
\end{gathered}
$$

где

$$
\begin{aligned}
\mathbf{f}_{\epsilon}(\alpha, \beta, \gamma) & =-\frac{\vartheta^{\prime}(0) \vartheta_{\epsilon}(\alpha) \vartheta_{\epsilon}(\beta) \vartheta_{\epsilon}(\beta-\alpha+2 \gamma)}{\vartheta_{\epsilon}(\alpha-\gamma) \vartheta_{\epsilon}(\beta+\gamma) \vartheta_{\epsilon}(\beta-\alpha+\gamma) \vartheta_{\epsilon}(\gamma)} \\
\wp_{\epsilon}(\gamma) & =\wp\left(\epsilon_{1} \gamma_{1}+\tau \epsilon_{2} \gamma_{2}\right), \quad \vartheta_{\epsilon}(\gamma)=\vartheta\left(\epsilon_{1} \gamma_{1}+\tau \epsilon_{2} \gamma_{2}\right)
\end{aligned}
$$

Доказательство предложения основано на спектральных свойствах функций $\varphi_{\epsilon}(\alpha, z)$.

Важным свойством линейных и квадратичных скобок является их согласованность. Она означает, что существует семейство скобок Пуассона, параметризованных $\lambda \in \mathbb{C} P^{1}$ :

$$
\{\mathbf{S}, \mathbf{S}\}_{\lambda}:=\lambda_{1}\{\mathbf{S}, \mathbf{S}\}_{1}+\lambda_{2}\{\mathbf{S}, \mathbf{S}\}_{2}, \quad \lambda=\frac{\lambda_{1}}{\lambda_{2}} .
$$

Чтобы доказать, что это выражение снова представляет собой скобку Пуассона, произведем замену $S_{0} \rightarrow S_{0}+\lambda$ в квадратичных скобках (3.21), (3.22). Тогда (3.21) не изменится, в то время как (3.22) приобретет дополнительный линейный член. Тем самым линейная комбинация $\{\mathbf{S}, \mathbf{S}\}_{\lambda}$ двух скобок может быть получена из квадратичной скобки простым сдвигом $S_{0}$. Результат сдвига, разумеется, по-прежнему удовлетворяет тождеству Якоби, а потому представляет собой скобку Пуассона при любом $\lambda$.

Существование семейства скобок Пуассона на $\mathbb{C} P^{1}$ приводит к бигамильтоновой структуре [8] эллиптической гидродинамики.

ПрЕДЛОЖЕНИЕ 4. Существует бесконечнъй набор сохраняющихся величин, коммутирующих между собой по отношению к обеим типам скобок,

$$
h_{s, j}, \quad j=0,2,3, \ldots, \quad s=0,1,2, \ldots,
$$

задающих бигамильтонову структуру иерархии

$$
\left\{h_{s, j}, \mathbf{S}\right\}_{1}=-\left\{h_{s+1, j}, \mathbf{S}\right\}_{2}
$$

Мы не приводим здесь интегралы $h_{s, j}$ в явном виде (см. [2]). Как частное следствие (3.27), поток (3.7) может быть представлен как

$$
\partial_{t} \mathbf{S}=\left\{S_{0}, \mathbf{S}\right\}_{2}
$$


3.5. Рациональный предел эллиптической гидродинамики. До сих пор рассматривалась только базисная спектральная кривая $\widetilde{E}_{\tau}$, параметризованная двумя полупериодами $\omega_{1}, \omega_{2}, \tau=\omega_{2} / \omega_{1}$. Заменим наши основные функции на

$$
\vartheta_{\epsilon}(\gamma)=\vartheta\left(\epsilon_{1} \gamma_{1} \omega_{1}+\epsilon_{2} \gamma_{2} \omega_{2} ; \omega_{1}, \omega_{2}\right), \quad \wp_{\epsilon}(\gamma)=\wp\left(\epsilon_{1} \gamma_{1} \omega_{1}+\epsilon_{2} \gamma_{2} \omega_{2} ; \omega_{1}, \omega_{2}\right)
$$

Теперь задача состоит в том, чтобы рассмотреть рациональный предел $\lim \omega_{1,2} \rightarrow \infty$ для $\widetilde{E}_{\tau}$. Исследуется двойной скейлинговый предел:

$$
\lim \epsilon_{1,2} \rightarrow 0, \quad \lim \omega_{1,2} \rightarrow \infty, \quad \lim \epsilon_{1} \omega_{1}=1, \quad \lim \epsilon_{2} \omega_{2}=\tau .
$$

Тогда $\lim \omega_{1,2} \rightarrow \infty$ приводит к рациональному вырождению эллиптических функций (3.28):

$$
\begin{gathered}
\lim _{\omega_{1,2} \rightarrow \infty} \vartheta_{\epsilon}(\gamma) \sim \gamma_{\tau}, \quad \lim _{\omega_{1,2} \rightarrow \infty} \wp_{\epsilon}(\gamma) \sim \frac{1}{\gamma_{\tau}^{2}}, \quad \gamma_{\tau}=\gamma_{1}+\gamma_{2} \tau, \\
\lim _{\omega_{1,2} \rightarrow \infty} \varphi_{\epsilon}(\alpha, z)=\left(\frac{1}{\alpha_{\tau}}+\frac{1}{z}\right), \quad \lim _{\omega_{1,2} \rightarrow \infty} f_{\epsilon}(\alpha, z)=-\frac{1}{\alpha_{\tau}^{2}} .
\end{gathered}
$$

Отсюда, в частности, следует, что обратный тензор инерции принимает вид

$$
\mathbf{J}(\mathbf{S})=\psi(x)=\partial_{\bar{Z}}^{-2} \mathbf{S}\left(x_{1}, x_{2}\right)=\sum_{\alpha \in \widetilde{\mathbb{Z}}^{(2)}} \frac{1}{\alpha_{\tau}^{2}} S_{-\alpha} T_{\alpha}, \quad \bar{Z}=\frac{2 \pi i}{\bar{\tau}-\tau}\left(\bar{\tau} x_{1}-x_{2}\right),
$$

где $\alpha_{\tau}=\alpha_{1}+\alpha_{2} \tau$. Уравнения движения задаются соответствующим квадратичным гамильтонианом

$$
H=-\frac{1}{2} \int_{T^{2}} \mathbf{S} \partial_{\bar{Z}}^{-2} \mathbf{S}=-\frac{1}{2} \sum_{\alpha \in \widetilde{\mathbb{Z}}^{(2)}} \frac{1}{\alpha_{\tau}^{2}} S_{\alpha} S_{-\alpha}
$$

по отношению к линейной скобке Пуассона на $\operatorname{Ham}^{0}\left(T^{2}\right)$

$$
\partial_{t} \mathbf{S}=\left\{\mathbf{S}, \partial_{\bar{Z}}^{-2} \mathbf{S}\right\}_{1}
$$

Последняя принимает следующий вид в фурье-компонентах:

$$
\partial_{t} S_{\alpha}=\sum_{\gamma \in \widetilde{\mathbb{Z}}^{(2)}} S_{\gamma} \frac{1}{\gamma_{\tau}^{2}} S_{\alpha-\gamma}
$$

Эти уравнения назовем модифицированной гидродинамикой на торе $T^{2}$.

Бездисперсионное уравнение Лакса

$$
\partial_{t} L\left(x_{1}, x_{2} ; z\right)=\left\{L\left(x_{1}, x_{2} ; z\right), M\left(x_{1}, x_{2}\right)\right\}_{1},
$$

где

$$
\begin{aligned}
L\left(x_{1}, x_{2} ; z\right) & =-\left(\partial_{\bar{Z}}^{-1}-\frac{1}{z}\right) \mathbf{S}\left(x_{1}, x_{2}\right), \\
M\left(x_{1}, x_{2}\right) & =-\partial_{\bar{Z}}^{-2} \mathbf{S}\left(x_{1}, x_{2}\right),
\end{aligned}
$$


эквивалентно уравнениям (3.30).

Бесконечный набор интегралов движения порождается разложением

$$
(-1)^{k} \int_{T^{2}} L^{k}\left(x_{1}, x_{2} ; z\right) d x_{1} d x_{2}=I_{0, k}+\sum_{m=0}^{k-2} I_{m, k} z^{-m-2} .
$$

Соответствующая бесконечная иерархия модифицированной гидродинамики $\partial_{s, k} \mathbf{S}=$ $\left\{I_{s, k}, \mathbf{S}\right\}_{1}$ допускает бездисперсионное представление Лакса.

В этом пределе выживает бигамильтонова структура. Имеет место следующая квадратичная алгебра Пуассона на торе $T^{2}$ :

$$
\begin{gathered}
\left\{S_{\alpha}, S_{0}\right\}_{2}=\sum_{\gamma \neq \alpha} S_{\alpha-\gamma} S_{\gamma} \frac{(\alpha \times \gamma)}{\gamma_{\tau}^{2}}, \\
\left\{S_{\alpha}, S_{\beta}\right\}_{2}=S_{0} S_{\alpha+\beta}(\alpha \times \beta)+\sum_{\gamma \neq \alpha,-\beta} S_{\alpha-\gamma} S_{\beta+\gamma} f(\alpha, \beta, \gamma)(\gamma \times(\alpha-\beta)),
\end{gathered}
$$

где

$$
f(\alpha, \beta, \gamma)=\left(\frac{1}{\gamma_{\tau}}+\frac{1}{\beta_{\tau}-\alpha_{\tau}+\gamma_{\tau}}-\frac{1}{\beta_{\tau}+\gamma_{\tau}}+\frac{1}{\alpha_{\tau}-\gamma_{\tau}}\right), \quad \gamma_{\tau}=\gamma_{1}+\tau \gamma_{2} .
$$

Иерархия модифицированной гидродинамики при этом снова описывается квадратичной алгеброй.

\section{4. АЛГЕБРОИД ЛИ ВЕКТОРНЫХ ПОЛЕЙ НА ТОРЕ}

4.1. Алгеброиды Ли и многообразия Пуассона. Начнем с краткого описания алгеброидов Ли. Детали этой теории содержатся в работах [9].

ОПРЕДЕЛЕНИЕ. Будем называть алгеброидом Ли над гладким многообразием $M$ векторное расслоение $\mathcal{A} \rightarrow M$

- со структурой алгебры Ли на пространстве его сечений $\Gamma(\mathcal{A})$, определяемой скобками Ли $\left\lfloor\varepsilon_{1}, \varepsilon_{2}\right\rfloor, \varepsilon_{1}, \varepsilon_{2} \in \Gamma(\mathcal{A})$;

- с отображением сечения (отображением якоря) $\delta: \Gamma(\mathcal{A}) \rightarrow T M$, удовлетворяющим следующим условиям:

1) для каждого $\varepsilon_{1}, \varepsilon_{2} \in \Gamma(\mathcal{A})$

$$
\left[\delta_{\varepsilon_{1}}, \delta_{\varepsilon_{2}}\right]=\delta_{\left\lfloor\varepsilon_{1} \varepsilon_{2}\right\rfloor},
$$

2) для каждого $\varepsilon_{1}, \varepsilon_{2} \in \Gamma(\mathcal{A})$ и $f \in C^{\infty}(M)$

$$
\left\lfloor\varepsilon_{1}, f \varepsilon_{2}\right\rfloor=f\left\lfloor\varepsilon_{1}, \varepsilon_{2}\right\rfloor+\left(\delta_{\varepsilon_{1}} f\right) \varepsilon_{2} .
$$

Другими словами, отображение якоря задает представление $\Gamma(\mathcal{A})$ в алгебру Ли векторных полей на $M$. Второе условие есть правило Лейбница по отношению к умножению сечений на гладкие функции. 
Пусть $\left\{e^{j}(x)\right\}$ - базис локальных сечений $\Gamma(\mathcal{A})$. Тогда скобки задаются структурными функциями $f_{i}^{j k}(x)$ алгеброида:

$$
\left\lfloor e^{j}, e^{k}\right\rfloor=f_{i}^{j k}(x) e^{i}, \quad x \in M .
$$

Приведем несколько примеров алгеброидов Ли.

1. Если отображение якоря тривиально, то $\mathcal{A}$ просто представляет собой расслоение алгебр Ли.

2. Второй пример менее тривиален (см. работы [4]). Пусть $M$ - многообразие Пуассона с бивектором Пуассона $\pi=\pi\left(\varepsilon, \varepsilon^{\prime}\right)$, где $\varepsilon, \varepsilon^{\prime}-$ сечения расслоения $T^{*} M$. Скобка Пуассона задается на пространстве гладких функций $\mathcal{H}(M)$ :

$$
\{f(x), g(x)\}:=\langle d f|\pi| d g\rangle=\partial_{k} f \pi^{k j} \partial_{j} g, \quad d f, d g \in T_{x}^{*} M .
$$

Бивектор Пуассона задает отображение

$$
V^{\pi}: T^{*} M \rightarrow T M, \quad V_{\varepsilon}^{\pi}=\langle\varepsilon|\pi| \partial\rangle=\varepsilon_{k} \pi^{k j} \partial_{j}
$$

Соответствующая производная Ли $\mathcal{L}_{V_{\varepsilon}}:=\delta_{\varepsilon}$ действует по правилу

$$
\delta_{\varepsilon}(x)=\pi(x)|\varepsilon\rangle .
$$

Таким образом, можно получить отображение из пространства гладких функционалов $\mathcal{H}(M)$ в пространство гамильтоновых векторных полей:

$$
f \rightarrow V_{f}=\langle d f|\pi| \partial\rangle
$$

Скобка Пуассона теперь может быть переписана в виде $\{f(x), g(x)\}=-i_{V_{d f}} d g$.

Можно задать скобку на один-формах $\varepsilon, \varepsilon^{\prime} \in \Gamma\left(T^{*} M\right)$ :

$$
\left\lfloor\varepsilon, \varepsilon^{\prime}\right\rfloor=d\left\langle\varepsilon|\pi(x)| \varepsilon^{\prime}\right\rangle+\left\langle d \varepsilon|\pi| \varepsilon^{\prime}\right\rangle+\left\langle\varepsilon|\pi| d \varepsilon^{\prime}\right\rangle .
$$

В координатном виде она задается выражением

$$
\left\lfloor\varepsilon, \varepsilon^{\prime}\right\rfloor_{k}=\partial_{k}\left(\varepsilon_{m} \pi^{m n} \varepsilon_{n}\right)+\partial_{k}\left(\varepsilon_{m}\right) \pi^{m n} \varepsilon_{n}+\varepsilon_{m} \pi^{m n} \partial_{k} \varepsilon_{n} .
$$

Из тождества Якоби для $\pi$ следует, что эта скобка является скобкой Ли. Более того, $\left[V_{\varepsilon}, V_{\varepsilon^{\prime}}\right]=V_{\left\lfloor\varepsilon, \varepsilon^{\prime}\right\rfloor}$. Таким образом, $T^{*} M$ наделяется структурой алгеброида Ли $\mathcal{A}_{\pi}$ над многообразием Пуассона $M$ со скобками Ли (4.7) и отображением якоря (4.4). Структурные функции на $T^{*} M$ задаются бивектором Пуассона $f_{i}^{j k}(x)=\partial_{i} \pi^{j k}(x)$.

Предположим теперь, что на $M$ заданы два совместных бивектора Пуассона $\pi_{1}$ и $\pi_{2}$,

$$
\pi(\lambda)=\lambda_{1} \pi_{1}+\lambda_{2} \pi_{2}
$$

где $\left(\lambda_{1}, \lambda_{2}\right)$ - однородные координаты на $\mathbb{C} P^{1}$. Бивектор Пуассона $\pi(\lambda)$ задает биалгеброид Ли $\mathcal{A}_{\pi(\lambda)}$ с помощью скобок (4.7) и соотношения якоря $(4.4)$, зависящего от $\lambda=\lambda_{1} / \lambda_{2} \in \mathbb{C} P^{1}$. 
4.2. Алгеброиды Ли векторных полей на торе. Нами уже определено семейство пуассоновых структур на пространстве $\operatorname{Ham}^{0 *}\left(T^{2}\right)(3.25)$ :

$$
\pi(\lambda) \sim\{\mathbf{S}, \mathbf{S}\}_{\lambda}=\{\mathbf{S}, \mathbf{S}\}_{2}+\lambda\{\mathbf{S}, \mathbf{S}\}_{1} .
$$

Они задают биалгеброид Ли $\mathcal{A}_{\pi(\lambda)}\left(T^{2}\right)$, база которого есть $\operatorname{Ham}^{*}\left(T^{2}\right)$, а сечения суть элементы $\operatorname{Ham}\left(T^{2}\right)^{3)}$

$$
\varepsilon(x)=\sum_{a \in \mathbb{Z} \oplus \mathbb{Z}} \varepsilon_{a} T_{a},
$$

где $\left\{T_{a}=\mathbf{e}(a \cdot x)\right\}-$ фурье-базис.

Исходя из формул (4.4) и (4.7), определим биалгеброид Ли:

$$
\begin{aligned}
\left\lfloor\varepsilon, \varepsilon^{\prime}\right\rfloor_{0}= & \sum_{\alpha, \beta \in \widetilde{\mathbb{Z}}^{2}} \varepsilon_{\alpha} \varepsilon_{\beta}^{\prime} S_{\alpha+\beta}(\alpha \times \beta), \\
\left\lfloor\varepsilon, \varepsilon^{\prime}\right\rfloor_{\gamma}= & \sum_{\alpha \in \widetilde{\mathbb{Z}}^{2}} \varepsilon_{\alpha}\left(\varepsilon_{0}^{\prime} S_{\alpha-\gamma} \wp_{\epsilon}(\gamma)+\lambda \varepsilon_{\gamma-\alpha}^{\prime}\right)(\alpha \times \gamma)+ \\
& +\sum_{\alpha, \beta \in \widetilde{\mathbb{Z}}^{2}} \varepsilon_{\alpha} \varepsilon_{\beta}^{\prime} S_{\alpha+\beta-\gamma} \mathbf{f}_{\epsilon}(\alpha, \beta, \alpha-\gamma)(\beta \times \alpha),
\end{aligned}
$$

где функции $\mathbf{f}_{\epsilon}, \wp_{\epsilon}$ определены в $(3.23),(3.24)$. Заметим, что структурные функции здесь имеют линейную зависимость от точки $\mathbf{S} \in \operatorname{Ham}^{0 *}\left(T^{2}\right)$. Постоянная составляющая структурных функций в (4.10) отвечает каноническим скобкам Пуассона на $T^{2}$. Представляется весьма интересным переписать это семейство скобок непосредственно в терминах векторных полей на торе $T^{2}$ с помощью соотношений между $\operatorname{Ham}^{0}\left(T^{2}\right)$ и $\operatorname{SVect}\left(T^{2}\right)$.

Как следует из (4.4), отображение якоря для биалгеброида $\mathcal{A}_{\pi(\lambda)}\left(T^{2}\right)$ имеет вид

$$
\begin{aligned}
\delta_{\varepsilon_{\alpha}} S_{0}= & \varepsilon_{\alpha}\left(\sum_{\gamma \neq \alpha} S_{\alpha-\gamma} S_{\gamma} \wp_{\epsilon}(\gamma)(\alpha \times \gamma)\right) \\
\delta_{\varepsilon_{\alpha}} S_{\beta}= & \varepsilon_{\alpha}\left(S_{0} S_{\alpha+\beta}(\alpha \times \beta)+\sum_{\gamma \neq \alpha,-\beta} S_{\alpha-\gamma} S_{\beta+\gamma} \mathbf{f}_{\epsilon}(\alpha, \beta, \gamma)(\gamma \times(\alpha-\beta))\right)+ \\
& +\lambda \varepsilon_{\alpha} S_{\alpha+\beta}(\alpha \times \beta) .
\end{aligned}
$$

\section{5. ЗАКЛЮЧЕНИЕ}

5.1. Некоммутативный тор. Можно непосредственно перейти от случая $T^{2}$ к некоммутативному тору $\mathcal{A}_{\theta}$. Для этого координаты $\left(x_{1}, x_{2}\right)$ следует заменить на операторы с коммутационными соотношениями $\left[x_{1}, x_{2}\right]=-2 \pi i \theta$. Некоммутативный тор представляет собой унитальную ассоциативную алгебру с двумя генераторами $\left(U_{1}, U_{2}\right)$, удовлетворяющими соотношению

$$
U_{1} U_{2}=\mathbf{e}_{\theta}^{-1} U_{2} U_{1}, \quad \mathbf{e}_{\theta}=e^{2 \pi i \theta}, \quad \theta \in[0,1) .
$$

3) Так как в определение квадратичной скобки был включен константный член $S_{0}$, можно перейти от $\operatorname{Ham}^{0 *}\left(T^{2}\right)$ к $\operatorname{Ham}^{*}\left(T^{2}\right)$. 
Алгебра $\mathcal{A}_{\theta}$ заменяет собой коммутативную алгебру $C^{\infty}\left(T^{2}\right)$ с помощью отождествления $U_{1} \rightarrow \mathbf{e}(x), U_{2} \rightarrow \mathbf{e}(y)$, где умножение в $\mathcal{A}_{\theta}$ задается умножением Мойала:

$$
(f * g)(x, y):=f g+\sum_{n=1}^{\infty} \frac{(\pi \theta)^{n}}{n !} \varepsilon_{r_{1}, s_{1}} \ldots \varepsilon_{r_{n}, s_{n}}\left(\partial_{r_{1} \ldots r_{n}}^{n} f\right)\left(\partial_{s_{1} \ldots s_{n}}^{n} g\right) .
$$

Вместо базиса Фурье (3.2) следует использовать базис

$$
T_{\alpha}=\frac{i}{2 \pi \theta} \mathbf{e}_{\theta}^{\alpha_{1} \alpha_{2} / 2} U_{1}^{m} U_{2}^{n}, \quad \alpha \in \widetilde{\mathbb{Z}}^{(2)},
$$

с коммутаторами

$$
\left[T_{\alpha}, T_{\beta}\right]=\mathbf{C}_{\theta}(\alpha, \beta) T_{\alpha+\beta}, \quad \mathbf{C}_{\theta}(\alpha, \beta)=\sin 2 \pi \theta\left(\alpha_{1} \beta_{2}-\alpha_{2} \beta_{1}\right),
$$

которые заменяют скобки Пуассона (4.6). Это приводит к определению бесконечномерной алгебры Ли (sin-алгебры) с коммутационными соотношениями (5.3); sinалгебра играет роль $\operatorname{Ham}\left(T^{2}\right)$.

Функционал следа $\operatorname{tr} f=f_{0}$ на $A_{\theta}$ удовлетворяет соотношению $\operatorname{tr}(f * g)=\operatorname{tr}(g * f)$, и с его помощью можно определить дуальное пространство $\mathcal{A}_{\theta}^{*}$. Завихренность на некоммутативном торе есть элемент $\mathcal{A}_{\theta}^{*}$, разложенный по базису $T_{\alpha}$ :

$$
\mathbf{S}=\sum S_{\alpha} T_{\alpha}
$$

Структура Пуассона-Ли на $\mathcal{A}_{\theta}^{*}$ задается выражением (5.3). Уравнения движения эллиптической гидродинамики на некоммутативном торе принимают вид

$$
\partial_{t} S_{\alpha}=\sum_{\gamma \in \widetilde{\mathbb{Z}}^{(2)}} S_{\gamma \wp_{\theta, \epsilon}(\gamma) S_{\alpha-\gamma}}
$$

где $\wp_{\theta, \epsilon}(\gamma)=\wp\left(\left(\epsilon_{1} \gamma_{1}+\epsilon_{2} \gamma_{2} \tau\right) \theta\right)$. Эти уравнения допускают представление Лакса $\partial_{t} L=[L, M]$, где коммутатор понимается в смысле $\sin -$ алгебры,

$$
L(x ; z)=-\sum_{\alpha \in \tilde{\mathbb{Z}}^{(2)}} S_{\alpha} \varphi_{\theta, \epsilon}(\gamma) T_{\alpha},
$$

и $\varphi_{\theta, \epsilon}(\gamma)=\mathbf{e}\left(\epsilon_{2} \gamma_{2} z \theta\right) \phi_{\theta, \epsilon}(\gamma, z), \quad \phi_{\theta, \epsilon}(\gamma, z)=\phi\left(\left(\left(\gamma_{1} \epsilon_{1}+\gamma_{2} \epsilon_{2} \tau\right) \theta\right), z\right) . \quad M$-оператор должен быть модифицирован соответствующим образом.

Рассмотрим теперь скобки Пуассона на $\mathcal{A}_{\theta}^{*}$. Правые части в определениях линейной и квадратичной алгебр Пуассона (3.17) и (3.20) становятся коммутаторами. В итоге квадратичная алгебра Пуассона сохраняет свой вид (3.33), (3.34):

$$
\begin{aligned}
\left\{S_{\alpha}, S_{0}\right\}_{2} & =\sum_{\gamma \neq \alpha} S_{\alpha-\gamma} S_{\gamma}\left(\wp_{\theta, \epsilon}(\gamma)-\wp_{\theta, \epsilon}(\alpha-\gamma)\right) \mathbf{C}_{\theta}(\alpha, \gamma), \\
\left\{S_{\alpha}, S_{\beta}\right\}_{2} & =S_{0} S_{\alpha+\beta} \mathbf{C}_{\theta}(\alpha, \beta)+\sum_{\gamma \neq \alpha,-\beta} S_{\alpha-\gamma} S_{\beta+\gamma} \mathbf{f}_{\theta, \epsilon}(\alpha, \beta, \gamma) \mathbf{C}_{\theta}(\gamma, \alpha-\beta),
\end{aligned}
$$

где

$$
\mathbf{f}_{\theta, \epsilon}(\alpha, \beta, \gamma)=-\frac{\vartheta^{\prime}(0) \vartheta_{\theta, \epsilon}(\alpha) \vartheta_{\theta, \epsilon}(\beta) \vartheta_{\theta, \epsilon}(\beta-\alpha+2 \gamma)}{\vartheta_{\theta, \epsilon}(\alpha-\gamma) \vartheta_{\theta, \epsilon}(\beta+\gamma) \vartheta_{\theta, \epsilon}(\beta-\alpha+\gamma) \vartheta_{\theta, \epsilon}(\gamma)}
$$


и $\vartheta_{\theta, \epsilon}=\vartheta_{\theta, \epsilon}(\gamma)=\vartheta\left(\left(\epsilon_{1} \gamma_{1}+\epsilon_{2} \gamma_{2} \tau\right) \theta\right)$.

Линейная и квадратичная скобки Пуассона совместны и задают бигамильтонову иерархию эллиптической гидродинамики на некоммутативном торе. Детали можно найти в работе [2].

5.2. Дальнейшее квантование. Квантование квадратичной алгебры Пуассона (5.4), (5.5) на некоммутативном торе приводит к ассоциативной алгебре с квадратичными соотношениями. Постоянная Планка $ћ$ принадлежит при этом эллиптической кривой $E_{\tau}$. Заменяя классические переменные $\mathbf{S}$ на операторы $\widehat{\mathbf{S}}$, получим, что

$$
\sum_{c} \widehat{S}_{a-c} \widehat{S}_{b+c} \mathbf{e}_{\theta}(c \times(a-b)) \mathbf{f}_{\theta, \epsilon}(a, b, c \mid \hbar)=0
$$

для любых $a, b \in \mathbb{Z} \oplus \mathbb{Z}$, где

$$
\mathbf{f}_{\theta, \epsilon}(a, b, c \mid \hbar)=-\frac{\vartheta^{\prime}(0) \vartheta_{\theta, \epsilon}(a+2 \hbar) \vartheta_{\theta, \epsilon}(b) \vartheta_{\theta, \epsilon}(b-a+2 c)}{\vartheta_{\theta, \epsilon}(a-c+\hbar) \vartheta_{\theta, \epsilon}(b+c+\hbar) \vartheta_{\theta, \epsilon}(b-a+c+\hbar) \vartheta_{\theta, \epsilon}(c+\hbar)} .
$$

Эти квадратичные соотношения представляют собой бесконечномерное обобщение квантовых алгебр Фейгина-Одесского.

Благодарности. Работа была частично поддержана РФФИ (гранты № 06-0217381 и НШ-8065-2006.2).

\section{Список литературы}

[1] С. Л. Зиглин, Докл. АН, 250:6 (1980), 1296-1300.

[2] B. Khesin, A. Levin, M. Olshanetsky, Commun. Math. Phys., 250 (2004), 581-612; nlin.SI/0309017.

[3] Е.К. Склянин, Функи. анализ и его прилож., 16:4 (1982), 27-34; А. В. Одесский, Б. Л. Фейгин, Функи. анализ и его прилож., 23:3 (1989), 45-54.

[4] М. В. Карасев, Изв. АН СССР. Сер. матем., 50:3 (1986), 508-538; В. Fuchssteiner, Progr. Theoret. Phys., 68 (1982), 1082-1104.

[5] А. В. Белавин, В. Г. Дринфельд, Функи. анализ и его прилож., 16:3 (1982), 1-29.

[6] V. I. Arnold, B. A. Khesin, Topological Methods in Hydrodynamics, Appl. Math. Sci., 125, Springer, N. Y., 1998.

[7] В. И. Арнольд, УМН, 24:3 (1969), 225-226.

[8] F. Magri, J. Math. Phys., 19 (1978), 1156-1162.

[9] K. Mackenzie, Lie Groupoids and Lie Algebroids in Differential Geometry, London Math. Soc. Lect. Notes Ser., 124, Cambridge Univ. Press, Cambridge, 1987; A. Cannas da Silva, A. Weinstein, Geometric Models for Noncommutative Algebras, Berkeley Math. Lect. Notes, 10, Amer. Math. Soc., Providence, RI; Berkeley Center for Pure and Appl. Math., Berkeley, CA, 1999. 